\title{
LATE-ONSET HEXOSAMINIDASE A DEFICIENCY MIMICKING PRIMARY LATERAL SCLEROSIS
}

\author{
Clecio Godeiro-Junior, Andre C. Felicio, Vinicius Benites, \\ Marco Antonio Chieia, Acary S.B. Oliveira
}

The GM2 gangliosidosis are a group of metabolic disorders in which deficiency of a lysosomal enzyme, hexosaminidase A (Hex A), leads to an abnormal intracellular accumulation of lipids in neurons and glia. Total deficiency is responsible for a fatal infantile disorder, Tay-Sachs disease, characterized by involution in motor abilities, hypotonia, seizures and cortical blindness, with death around 5 or 6 years-old. Partial deficiency of enzyme activity is associated with a variety of late-onset (teenagers and young adults) neurological phenotypes, characterized by upper and lower motor neuron signs, cerebellar disturbances, parkinsonism, and psychosis or dementia in different combinations $^{1,2}$. Macular cherry red spots, which are typical for Tay-Sachs, are not apparent in these patients. All of the GM2 gangliosidosis subtypes are inherited in an autosomal recessive fashion and the differences in age at onset and disease course may be partly attributed to the underlying Hex A gene defect. Patients with infantile onset tend to be homozygous or compound heterozygote for severe, deleterious ("null") alleles, whereas patients with late-onset disease have a combination of one severe and another allele associated with residual Hex A activity ${ }^{3}$. Late onset GM2 gangliosidosis (LOGM2) is commonly included in the differential diagnosis of amyotrophic lateral scle- rosis (ALS), due to the involvement of anterior horn cell, causing weakness, atrophy, cramps and fasciculations ${ }^{3,4}$.

We report a patient with unusual clinical features of isolated upper motor neuron (UMN) involvement, resembling primary lateral sclerosis (PLS).

\section{CASE}

A 30-year-old Brazilian Caucasian man was referred to our department for neurological assessment due to an 8-year history of progressive weakness involving the right side of his body. Insidiously, the symptoms progressed to his left leg and arm. He developed dysarthria and dysphagia. He also noted difficulty for recalling recent events. His past medical history was unremarkable. Family history was negative for neurological disorders and his parents were not consanguineous.

On neurological evaluation, his cognition was preserved. Neurological examination disclosed spastic tetraparesis (MRC $4 / 5$ in all limbs) with global hyperreflexia, including bilateral Babinski and Hoffman signs. There was bilateral VI nerve palsy, but examination with ophthalmoscope was normal. The patient did not present sphincterian damage.

Brain magnetic resonance imaging (MRI) showed a hyperintense signal along the pyramidal tract in FLAIR (Figure). Electroneuromyography (ENMG) did not show signs of denervation

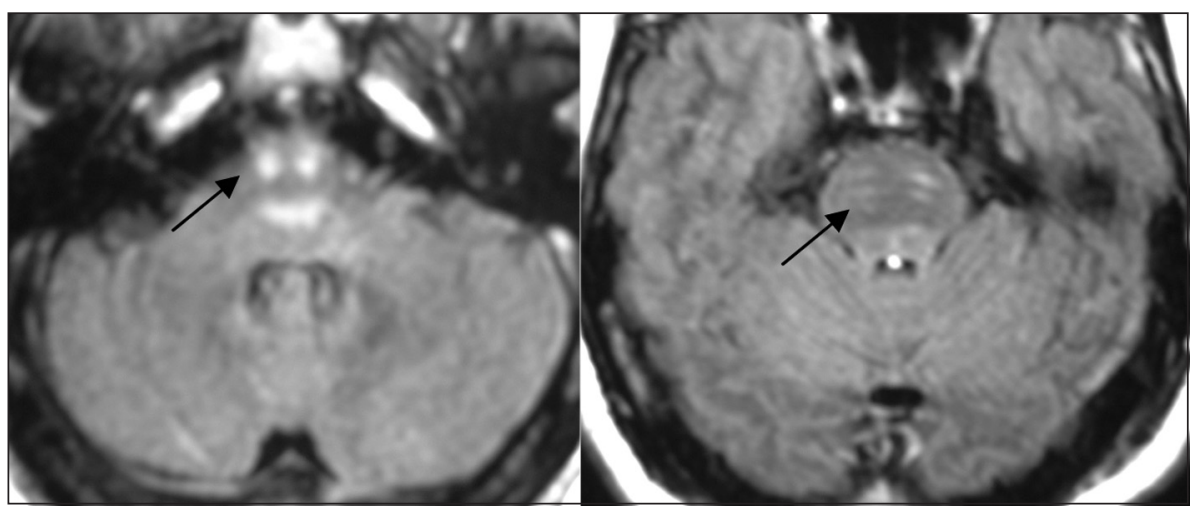

Figure. MRI showing a hyperintense signal along the pyramidal tract in FLAIR (Black arrows).

\section{DEFICIÊNCIA TARDIA DE HEXOSAMINIDASE-A SIMULANDO ESCLEROSE LATERAL PRIMÁRIA}

Neuromuscular Disorders Unit, Department of Neurology and Neurosurgery, Escola Paulista de Medicina, Federal University of São Paulo, São Paulo SP, Brazil.

Received 24 June 2008, received in final form 17 October 2008. Accepted 12 November 2008

Dr. Clecio Godeiro-Junior - Rua Dr. Diogo de Faria, 650 / 33 - 04037-002 São Paulo SP - Brasil. E-mail: cleciojunior@yahoo.com.br 
or reinervation, nerve conduction was normal, but there was an increased insertional activity on tested muscles. Although clinical manifestations were similar to PLS, impairment of extraocular muscles and early onset impelled further investigation. The cerebrospinal fluid examination was normal, such as standard hematologic and biochemical tests: full blood count, erythrocyte sedimentation rate, glucose, urea and creatinine, electrolytes, liver function, thyroid function, B12 vitamin, ANA. Serology for, HIV, HTLV-1, VDRL, hepatitis B and C were negative. The patient sera was also analyzed for hexosaminidase $A$ by a fluorometric method: Hex A - 280 nmoles $/ \mathrm{h} / \mathrm{mL}$ (normal value: 550-1.675) and $32 \%$ of total hexosaminidase (normal value: $45-$ $70 \%$ ). Due to these results, we concluded that patient's signs and symptoms were due to partial Hex A deficiency.

Despite symptomatic treatment with baclofen the patient did not improve and is currently on follow-up.

An informed consent was signed allowing data publication.

\section{DISCUSSION}

LOGM2 is rarely a potential cause for ALS-mimic syndromes, and most commonly LMN symptoms are predominant $^{5}$. Early findings include decreased or absent triceps and patellar reflexes, an easy startle, primarily to light touch when unexpected, hand tremors, fasciculations, and a positive palmomentum reflex ${ }^{3}$. Our patient presented a progressive spastic tatra paresis, resembling PLS, which is uncommon for LOGM2. Moreover, brain MRI findings revealed abnormal signal on corticospinal tract, as seen at typical ALS, and no other abnormalities ${ }^{6}$. This finding was uncommon, once brain imaging studies in patients with LOGM2 generally show severe cerebellar atrophy ${ }^{7}$.

PLS is another rare disorder, characterized by a slowly progressive symmetrical pyramidal deficit occurring in the absence of muscle wasting and in which no other cause for the neurological dysfunction can be established ${ }^{8}$. The differential diagnosis for progressive corticospinal spasticity is challenging. Many disorders, such as inherited illnesses or conditions with sensory symptoms or more generalized neurologic impairment, could be eliminated readily after a careful history and examination. Actually, if a patient presents with slowly progressive spinobulbar spasticity in the absence of other findings, the differen- tial diagnosis is limited. The hereditary spastic paraplegias and familial ALS merit particular consideration ${ }^{4}$. If the patient is less than 40 years of age, Hex A levels could be of value to diagnose LOGM2, which may be quite difficult to distinguish from ALS on clinical examination. Although the motor manifestations of our patient were typical of a MND, some associated findings impelled us to find an alternative diagnosis: early manifestation, slow course and bilateral VI nerve palsy. The clinical features described in patients with Hex A deficiency and motor neuron symptoms include early onset, positive family history, and/or long disease duration ${ }^{1-3,5}$.

Previous reports failed to reveal significant Hex A deficiency among ALS patients. In one study, the frequency of mutations in the Hex A gene among Israeli ALS patients was not higher than in the healthy Israeli population ${ }^{5}$. In another one, Hex A activity determined in isolated peripheral blood leukocytes was screened in ALS patients, and was normal in all typical ALS patients ${ }^{9}$. In both studies there is no data regarding PLS.

This case report highlights that Hex A deficiency can mimic not only ALS, but also PLS. We believe that the screening for Hex A deficiency should always be considered in atypical cases of MND.

\section{REFERENCES}

1. Navon R, Argov Z, Frisch A. Hexosaminidase A deficiency in adults. Am J Med Genet 1986;24:179-196.

2. Shapiro BE, Logigian EL, Kolodny EH, Pastores GM. Late-onset TaySachs disease: the spectrum of peripheral neuropathy in 30 affected patients. Muscle Nerve 2008;38:1012-1015.

3. Neudorfer O, Pastores GM, Zeng BJ, Gianutsos J, Zaroff CM, Kolodny $\mathrm{EH}$. Late-onset Tay-Sachs disease: phenotypic characterization and genotypic correlations in 21 affected patients. Genet Med 2005;7:119-123.

4. Baek WS, Desai NP. ALS: pitfalls in the diagnosis. Pract Neurology 2007;7:74-81

5. Drory VE, Birnbaum M, Peleg L, Goldman B, Korczyn AD. Hexosaminidase A deficiency is an uncommon cause of a syndrome mimicking amyotrophic lateral sclerosis. Muscle Nerve 2003;28:109-112.

6. Rocha AJ, Oliveira AS, Fonseca RB, Maia AC Jr, Buainain RP, Lederman HM. Detection of corticospinal tract compromise in amyotrophic lateral sclerosis with brain MR imaging: relevance of the T1-weighted spinecho magnetization transfer contrast sequence. AJNR Am J Neuroradiol 2004;25:1509-1515.

7. Streifler JY, Gornish M, Hadar H, Gadoth N. Brain imaging in late-onset GM2 gangliosidosis. Neurology 1993;43:2055.

8. Singer MA, Statland JM, Wolfe GI, Barohn RJ. Primary lateral sclerosis. Muscle Nerve 2007;35:291-302.

9. Gudesblatt M, Ludman MD, Cohen JA, et al. Hexosaminidase A activity and amyotrophic lateral sclerosis. Muscle Nerve 1988;11:227-230. 